\title{
Análise de eficiência dos gastos públicos em saúde nos municípios paranaenses
}

\author{
Analysis of the efficiency of public spending on health in the Paraná's municipalities \\ Análisis de eficiencia del gasto público en salud en los municipios de Paraná
}

Recebido: 25/01/2022 | Revisado: 02/02/2022 | Aceito: 05/02/2022 | Publicado: 09/02/2022

\author{
Vanderléia de Souza da Silva \\ ORCID: https://orcid.org/0000-0003-0525-9137 \\ Universidade Estadual de Campinas, Brasil \\ E-mail: vanderleia_adm@hotmail.com \\ Johan Hendrik Poker Junior \\ ORCID: https://orcid.org/0000-0001-7086-8786 \\ Universidade Estadual de Campinas, Brasil \\ E-mail: johanpkr@unicamp.br \\ Maria de Nazareth Rodrigues Malcher de Oliveira Silva \\ ORCID: https://orcid.org/0000-0003-4405-7378 \\ Universidade de Brasília, Brasil \\ E-mail: malchersilva@unb.br
}

\begin{abstract}
Resumo
A falta da gestão eficiente dos gastos públicos em saúde afeta os cidadãos principalmente no seu desempenho, em educação e no trabalho, e no desenvolvimento humano. Além disso, existe uma preocupação global com os gastos públicos, principalmente em saúde, devido à escassez de recursos na esfera pública. Logo, o objetivo deste estudo foi analisar a eficiência dos municípios paranaenses na gestão dos gastos públicos em saúde e saneamento e a variação do IDH-M fator longevidade, buscando identificar variáveis intervenientes no período de 2000-2009. Para isso, foi utilizada a Análise Envoltória de Dados (DEA) e a Regressão Linear Múltipla. Os resultados apontaram cinco municípios eficientes e os fatores intervenientes identificados como interventores à eficiência foram: a distância da capital estadual; o número de ocorrência de neoplasias na população; o município ser sede de regional de saúde e a variação do IDH-M fator Educação. A contribuição do estudo reside na identificação dos fatores intervenientes à gestão do gasto público em saúde, pois o tratamento destes fatores pode aumentar a longevidade dos munícipes. E ainda, foi identificado que o aumento dos gastos públicos em saúde e saneamento não se faz necessário, em alguns dos municípios.
\end{abstract}

Palavras-chave: DEA-dois estágios; Gestão pública; Recursos em saúde.

\begin{abstract}
The lack of efficient management of public spending on health affects citizens primarily in their performance, in education and work, and in human development. Moreover, there is a global concern about public spending, especially in health, due to the scarcity of resources in the public sphere. Therefore, the objective of this study was to analyze the efficiency of Paraná municipalities in the management of public spending on health and sanitation and the variation of the HDI-M longevity factor, seeking to identify intervening variables in the period 2000-2009. For this, Data Envelopment Analysis (DEA) and Multiple Linear Regression were used. The results indicated five efficient municipalities and the intervening factors identified as intervening in the efficiency were: distance from the state capital; the number of occurrences of neoplasms in the population; the municipality being the headquarters of a health regional office, and the variation of the HDI-M Education factor. The contribution of the study lies in the identification of factors that intervene in the management of public spending in health, since the treatment of these factors can increase the longevity of the population. And yet, it was identified that increasing public spending on health and sanitation is not necessary, in some of the municipalities.
\end{abstract}

Keywords: DEA-two stage; Health resources; Public management.

\section{Resumen}

La falta de gestión eficiente del gasto público en salud afecta a los ciudadanos principalmente en su rendimiento, en la educación y el trabajo, y en el desarrollo humano. Además, existe una preocupación global por el gasto público, especialmente en sanidad, debido a la escasez de recursos en el ámbito público. Por lo tanto, el objetivo de este estudio fue analizar la eficiencia de los municipios de Paraná en la gestión del gasto público en salud y saneamiento y la variación del factor de longevidad del IDH-M, buscando identificar variables intervinientes en el período 2000 2009. Para ello, se utilizó el Análisis Envolvente de Datos (DEA) y la Regresión Lineal Múltiple. Los resultados apuntan a cinco municipios eficientes y los factores de intervención identificados como factores de eficiencia son: la distancia a la capital del estado, el número de neoplasias en la población, el hecho de que el municipio sea sede de una 
región de salud y la variación del IDH-M en la educación. La contribución del estudio radica en la identificación de los factores que intervienen en la gestión del gasto público en salud, ya que el tratamiento de estos factores puede aumentar la longevidad de los residentes. Además, se ha detectado que el aumento de los gastos públicos en salud y sanidad no es necesario en algunos municipios.

Palabras clave: DEA-dos etapas; Gestión pública; Recursos en salud.

\section{Introdução}

O efeito da falta de saúde na vida do cidadão é como um ciclo, por exemplo, quando há falta saúde o indivíduo não consegue estudar e trabalhar e, em consequência disso, não obtêm maior renda, pois sem educação o indivíduo não se aperfeiçoa para ocupar um posto de trabalho melhor, e ainda, deixa de direcionar tempo para outras oportunidades e capacidades. Então, a promoção da saúde é uma estratégia que, por meio de ações generalizadas, busca transformações nas pessoas a fim de que exerçam seus direitos e sintam-se cidadãos (Carrara \& Ventura, 2012).

Existe uma preocupação global com os gastos públicos, principalmente em saúde, pois estes recursos estão cada vez mais escassos, devido ao aumento do número de usuários dos sistemas públicos de saúde, do aumento da incidência de doenças que têm alto custo e das irregularidades na gestão dos recursos. Em 2014, o Brasil teve o terceiro maior gasto total per capita em saúde na América Latina, representando quase 950 dólares, ficando atrás do Chile e Uruguai, segundo dados do World Health Organization [WHO] (2017). Mas quando estes valores são comparados à expectativa de vida nos países da América Latina, o Paraguai e o Peru, que gastaram menos da metade do que o Brasil alcançou índices similares de longevidade, demonstrando que o montante gasto nem sempre está relacionado com a eficiência.

As evidências científicas mostradas abundantemente na literatura indicam a contribuição da saúde para a qualidade de vida dos indivíduos e populações (Buss, 2000). Assim, a importância de estudos em saúde se dá pelo efeito causado no bem estar da população (Lepchack et al., 2021), que consequentemente pode aumentar ou reduzir a longevidade. Por isso, a falta da gestão eficiente dos gastos públicos em saúde baseada nas necessidades dos cidadãos afeta principalmente as áreas da: educação, trabalho, renda e no desenvolvimento humano.

O sistema de saúde brasileiro inicialmente atendia as necessidades sanitárias da região central do país, evoluindo à descentralização federal da gestão de recursos públicos em saúde para as demais esferas de governo a partir de 1988 (Lepchack et al., 2021). Com isso, os municípios passaram a ter maior responsabilidade sobre o gasto público em saúde e sobre a captação de recursos nas esferas estadual e federal. Nesse sentido, passaram a desempenhar um papel mais ativo no atendimento das necessidades de saúde da população local (Campos et al., 2016).

Segundo Dias (2016), o desperdício do dinheiro público ainda é considerado alto frente aos serviços dispostos à população. Esta realidade pode ser vista em diversos municípios paranaenses que possuem gestores públicos (prefeitos, vereadores e secretários municipais) envolvidos em esquemas de corrupção (desvios de verba, pactuação de contratos superfaturados, contratação de mão-de-obra irregular, distribuição de remédios vencidos, entre outros). Dias (2016) analisou os fatores que impactam na corrupção e na ineficiência relacionadas à aplicação de recursos da saúde pública municipal brasileira, e concluiu que a governança pública e o ambiente propício ao comportamento rent-seeking são fatores que impactam na corrupção e na eficiência relacionadas à aplicação dos recursos municipais. Concluiu ainda que uma maior fiscalização por parte dos Conselhos Municipais de Saúde (CMS) e uma melhor gestão fiscal aumentam a chance de um município ser eficiente no gasto com saúde pública.

Abordando a questão de eficiência em saúde, sabe-se que cada procedimento gera diferentes custos e como o objetivo é salvar vidas e os recursos são finitos, deve-se atentar para que estes recursos não caiam através das "rachaduras", pois o desperdício é um luxo que não pode ser pago por nenhum sistema de saúde no mundo. Assim, a relação meios-fins tem quatro dimensões: eficácia médica (tratamento adequado para garantir o resultado); eficácia econômica (menor comprometimento dos 
recursos sociais); custo-benefício (comparação e avaliação dos alvos); e custo-utilidade (satisfação sentida pela sociedade) e o uso prudente dos recursos faz com que o sistema de saúde possa avançar no quesito eficiência, afirma Reisman (2007).

No Brasil, o estado do Paraná é um dos mais ativos na economia do país, representou 6\% do Produto Interno Bruto (PIB) nacional em 2010, e concentra uma alta produção de alimentos, veículos e madeira e subprodutos (Secretaria Estadual de Saúde, 2017). Mesmo o estado tendo uma economia forte e alta arrecadação de impostos, existem disparidades nos gastos públicos em saúde e saneamento entre os municípios. Como exemplo, têm-se os municípios de Foz do Iguaçu e Cascavel, que possuíam uma população em torno de 250 mil habitantes em 2010, e o valor do gasto público total entre 2000 e 2009 foi de pouco mais de R \$ 646 milhões em Foz do Iguaçu e de quase R \$ 376 milhões em Cascavel, conforme dados das Finanças do Brasil (Finbra, 2017).

Com base na desproporcionalidade do gasto público em saúde e saneamento no Paraná e a falta de estudos recentes sobre esta problemática no estado, despertou-se o interesse em elaborar um estudo sobre a eficiência da gestão. Além disso, é importante a identificação de fatores intervenientes à gestão pública para que auxiliem o gestor na elaboração de política pública e tomada de decisão. Assim, o objetivo deste estudo é analisar a eficiência dos gastos públicos em saúde e saneamento realizados em 363 municípios paranaenses perante a longevidade, no período de 2000 a 2009, e identificar as variáveis intervenientes na gestão pública. Para isso, serão aplicadas as metodologias de Análise Envoltória de Dados (Data Envelopment Analysis - DEA) e Regressão Linear Múltipla.

O estudo está organizado em quatro seções, sendo a primeira com a introdução e revisão de literatura, abordando a metodologia DEA e os estudos que aplicam o método na análise da eficiência do gasto público em saúde. A segunda parte dispõe sobre os procedimentos metodológicos, seguida das aplicações e resultados destes na seção quatro. E por fim, apresentam-se as considerações finais do estudo, limitações e sugestões para pesquisas futuras.

\subsection{Análise Envoltória de Dados (DEA) e aplicações}

As organizações utilizam métodos para avaliar seus desempenhos internos e externos, geralmente com uso de dados. Através do benchmarking, as organizações podem reconhecer as melhores práticas e aplicá-las na própria organização de forma adaptada às suas necessidades, servindo tanto para empresas públicas quanto para as privadas. Assim, as organizações avaliam sua produtividade, que conceitualmente surge da razão entre outputs e inputs. Os inputs são as entradas do processo, representando os recursos utilizados no processo de transformação do produto/serviço; e os outputs são as saídas, ou seja, os resultados dos processos realizados.

Quando se refere à produtividade, todos os fatores produtivos devem ser levados em consideração, não somente o resultado da produção. Produtividade e eficiência são distintas, a primeira considera a relação entre as entradas e saídas para a produção, já a segunda reflete o estado da tecnologia da empresa representando se ela está tecnicamente eficiente, ou abaixo da fronteira de eficiência (Andersen \& Pettersen, 1996; Coelli et al., 2005).

Baseado no conceito produtivo e na busca pelos benchmarkings, Charnes, Cooper e Rhodes (1978) desenvolveram a metodologia DEA, com um modelo matemático denominado Retornos Constantes de Escala (Constant Returns to Scale CRS), através da programação linear, para analisar a eficiência das unidades tomadoras de decisão (Decision Making Units $D M U$ ). O modelo DEA-CRS mede a eficiência com a maximização das saídas ponderadas pelas entradas ponderadas das DMUs (equação 1), sujeito a condição de que pesos similares de cada DMU sejam menores ou iguais a 1 para a unidade (equação 2), conforme representado na formulação de (Charnes et al., 1978):

$$
h_{0}=\frac{\sum_{r=1}^{s} u_{r} y_{r 0}}{\sum_{i=1}^{m} v_{i} x_{i 0}} h_{0}=\frac{\sum_{r=1}^{s} u_{r} y_{r 0}}{\sum_{i=1}^{m} v_{i} x_{i 0}}
$$




$$
\begin{gathered}
\text { sujeito } a: \frac{\sum_{r=1}^{s} u_{r} y_{r j}}{\sum_{i=1}^{m} v_{i} x_{i j}} \leq 1 ; j=1, \ldots, n . \\
v_{r} \geq 0 ; \quad r=1, \ldots, s ; \quad i=2, \ldots, m .
\end{gathered}
$$

O modelo assume que cada n DMU avaliada, consome m inputs e produz s outputs. Ou seja, cada DMUj consome a quantidade de xij de input (i) e produz a quantidade de yrj de outputs (r). Assim, cada DMU tem pelo menos uma entrada e uma saída positiva. E, urj = peso do output, e vrj = o peso do input (Seiford \& Thrall, 1990). Como as equações do modelo utilizavam a forma fracionária, na função objetivo e na restrição, não podiam ser resolvidas em programação linear, então os autores solucionaram o problema através da linearização.

O modelo matemático pode ser orientado aos outputs, que maximiza os níveis de produção atuais de saída, e orientado aos inputs, que visa reduzir os montantes de insumos pelo máximo possível (Cooper et al., 2002). Ambos apresentam como resultado a eficiência relativa das DMUs, sendo eficientes aquelas que atingem 1 (100\%) e as ineficientes são as que estão abaixo de 1 (Cooper et al., 2011).

Anos mais tarde, os autores Banker et al. (1984) desenvolveram o modelo DEA de Retornos Variáveis de Escala (Variable Returns to Scale - VRS), substituindo o axioma da proporcionalidade de inputs e outputs pelo axioma da convexidade. Assim, o modelo DEA-CRS foi adaptado para o DEA-VRS, com o acréscimo da restrição $\sum_{k=1}^{n} \quad \lambda_{k}=1 \sum_{k=1}^{n} \quad \lambda_{k}=1$ que garante a comparação de DMUs do mesmo tamanho.

A diferença entre os modelos DEA-CRS e DEA-VRS está nos retornos das escalas, sendo o primeiro constante e o segundo variável. Assim, se um aumento proporcional em um ou mais inputs causar um aumento maior que a proporção nos outputs, então o modelo DEA-CRS não pode ser aplicado, pois os retornos não estão sendo constantes. Da mesma forma, se um aumento proporcional dos inputs render menos do que o aumento proporcional nos outputs, a noção de variação fica caracterizada e o modelo DEA-VRS deve ser aplicado (Ozcan, 2008).

Além da escolha do modelo DEA e de sua orientação, para aplicação da metodologia DEA faz-se necessário seguir outras premissas, como a escolha de DMUs homogêneas; o conjunto de inputs e outputs; e os dados de inputs e outputs (Dyson et al., 2001).

Para medição da eficiência podem ser aplicadas diversas técnicas, sendo as mais aplicadas, além da DEA, Stochastic Frontier Analysis (SFA) e Free Disposal Hull (FDH). A diferença entre elas está, basicamente, na utilização de métodos paramétricos ou não-paramétricos; na formulação do modelo ser estatístico ou de programação; e no resultado que apresentam as empresas eficientes ou ineficientes. A DEA não traz apenas resultados de quais DMUs são eficientes e ineficientes, mas também apresenta os benchmarkings para melhoria do desempenho (Ozcan, 2008).

Segundo Liu et al. (2013), a importância da DEA está em sua capacidade de avaliar a eficiência individual ou o desempenho de uma unidade de tomada de decisão (DMU) dentro de um grupo de interesse alvo que opera e domina a aplicação em um setor específico, como bancário, saúde, agricultura, transportes, educação, etc. Ainda segundo os autores, a avaliação de eficiência em saúde está entre os cinco setores que mais aplicam DEA, ocupando a segunda posição com 39\% das publicações entre 2005 a 2009, ficando atrás apenas do setor bancário, com 45,5\% das publicações. Assim, verifica-se a grande aplicabilidade desta metodologia na avaliação de eficiência em saúde.

\subsubsection{Aplicações de DEA em gasto público em saúde}

Desde a publicação do primeiro estudo com a metodologia DEA em 1978, diversos outros estudos foram elaborados com contribuições, tanto na melhoria do método de cálculo quanto na ampliação da aplicação nas áreas de pesquisa. Finalidades de diferentes setores de atuação e propósitos surgiram, e mais especificamente, diferentes setores da economia 
foram favorecidos pelo uso do DEA na busca de melhores práticas com impacto em eficiência e produtividade (Cooper et al., 2011; Emrouznejad et al., 2008).

Consequentemente, na área da saúde também foram feitas aplicações e os três primeiros estudos, segundo Lui et al. (2013), foram elaborados por: Nunamaker (1993) que mensurou a eficiência no serviço de enfermagem; Sherman (1984) e Banker, Conrad e Strauss (1986), ambos mediram a eficiência hospitalar. Com o passar dos anos, outras abordagens aplicaram DEA na avaliação em saúde, como por exemplo, em saúde básica, casas de repouso, programas de saúde, etc. (Liu et al., 2013).

A DEA foi aplicada em estudos brasileiros somente no final do século XX, sendo que os primeiros trabalhos em saúde foram elaborados por: Zucchi (1998), que avaliou a eficiência de hospitais públicos e privados; Façanha e Marinho (1998), avaliaram a eficiência de hospitais universitários; e Marinho e Silva (1998), que analisaram o desempenho do sistema de saúde (Marinho, 2001).

Considerando os primeiros estudos até os dias atuais, a análise de eficiência dos hospitais ou dos serviços de saúde, sejam públicos ou privados, foram base para estudos em diversas nações (Santos, Souza Francisco \& Gonçalves, 2016). Com a divulgação de informações financeiras do setor público em bases de dados, novos estudos se debruçaram sobre a temática da eficiência do gasto público em saúde.

Na pesquisa bibliográfica realizada, verificou-se que os estudos encontrados no meio internacional sobre a eficiência do gasto público em saúde, estão, em sua maioria, voltados para a análise de eficiência do sistema de saúde de uma determinada região, considerando a variável de gasto público em saúde, geralmente, aplicada em valores per capita. Assim, apresenta-se uma breve revisão de algumas publicações que tem como objetivo principal analisar o gasto público em saúde através da metodologia DEA e aplicam uma variável financeira (compreendendo o gasto público em saúde).

$\mathrm{Na}$ Tabela 1, tem-se uma apresentação de estudos internacionais recentes focados na aplicação da DEA ao gasto público em saúde.

Tabela 1. Aplicação de DEA x Gasto Público em Saúde.

\begin{tabular}{|c|c|c|}
\hline Autor (es) & Objetivo e métodos & $\operatorname{Input}(\mathrm{s})$ e $\operatorname{output}(\mathrm{s})$ \\
\hline Hsu (2013) & $\begin{array}{l}\text { Avaliar a eficiência dos gastos governamentais de } 46 \text { países } \\
\text { em saúde, entre } 2005 \text { a } 2007 . \\
\text { DEA-Dois Estágios orientado aos outputs e Índice de } \\
\text { Malmquist e Regressão de Tobit. }\end{array}$ & $\begin{array}{l}\text { Input: gasto em saúde per capita. } \\
\text { Outputs: expectativa de vida ao nascer, taxa de } \\
\text { mortalidade infantil e taxa de imunização contra o } \\
\text { sarampo. }\end{array}$ \\
\hline Novignon (2015) & $\begin{array}{l}\text { Mensurar a eficiência das instituições públicas de saúde em } \\
45 \text { países desta região e o período coberto pelo estudo foi } \\
\text { de } 1995 \text { a } 2010 . \\
\text { DEA-Dois Estágios e Regressão de Tobit. }\end{array}$ & $\begin{array}{l}\text { Input: despesa per capita em saúde e média de } \\
\text { escolaridade. } \\
\text { Outputs: expectativa de vida, taxa de mortalidade } \\
\text { infantil e mortalidade de menores de cinco anos; e a } \\
\text { taxa bruta de mortalidade. }\end{array}$ \\
\hline et al. (2016) & $\begin{array}{l}\text { Analisar a eficiência dos recursos públicos investidos pelos } \\
17 \text { governos regionais da Espanha nos sistemas de saúde. } \\
\text { DEA-CRS e VRS orientados aos inputs. }\end{array}$ & $\begin{array}{l}\text { Inputs: despesas em saúde pública per capita e o } \\
\text { percentual de despesas trabalhistas. } \\
\text { Outputs: frequência de internamentos, frequência de } \\
\text { serviços especializados externos e serviços básicos } \\
\text { em medicina e enfermagem per capita. }\end{array}$ \\
\hline $\begin{array}{l}\text { Rocha, Duarte, } \\
\text { Oliveira, Pereira e } \\
\text { Gadelha (2017) }\end{array}$ & $\begin{array}{l}\text { Verificar a necessidade de aumento da quantidade ou } \\
\text { melhoria no uso dos recursos, em } 5.523 \text { municípios } \\
\text { brasileiros, com dados de } 2010 \text {. } \\
\text { CRS e VRS orientados aos inputs e Regressões Quantílicas. }\end{array}$ & $\begin{array}{l}\text { Inputs: gastos per capita em saúde e a escolaridade } \\
\text { das pessoas acima de } 25 \text { anos. } \\
\text { Output: Índice de Desempenho do SUS (IDSUS). }\end{array}$ \\
\hline $\begin{array}{l}\text { Lepchack et al. } \\
(2021)\end{array}$ & $\begin{array}{l}\text { Analisar a eficiência dos recursos destinados à saúde } \\
\text { pública nos } 76 \text { maiores municípios brasileiros, no período } \\
\text { de } 2013 \text { a } 2017 \text {. } \\
\text { VRS orientado aos inputs e correlação. }\end{array}$ & $\begin{array}{l}\text { Inputs: gastos públicos em saúde, atenção básica, } \\
\text { assistência hospitalar e ambulatorial, suporte } \\
\text { profilático e terapêutico, vigilância sanitária e } \\
\text { epidemiológica, alimentação e nutrição. } \\
\text { Output: Autorização de internação hospitalar, óbitos, } \\
\text { dias de permanência, valor médio das internações e } \\
\text { serviços hospitalares e dias de internação. }\end{array}$ \\
\hline
\end{tabular}

Nota: Resumo de estudos que aplicaram a metodología DEA para mensuração de eficiência. Fonte: Adaptado de Hsu (2013), Novignon (2015), Campos et al. (2016), Rocha et al. (2017) e Lepchack et al. (2021). 
No Brasil, a DEA tem sido aplicada para a comparação da eficiência em diversos setores. Uma das áreas de aplicação que mais podem beneficiar-se da aplicação da DEA é o sistema público de saúde. Apesar das melhorias constantes no sistema de saúde brasileiro, principalmente na oferta dos serviços, a área ainda carece de estudos que permitam que práticas gerenciais inovadoras sejam identificadas e reproduzidas de forma mais sistemática. Uma vez que, a maioria dos estudos dispostos na literatura avalia apenas a quantidade ou o tipo de serviço prestado frente aos recursos gastos, sem considerar a efetividade destes serviços na longevidade da população. Ou seja, a gestão dos recursos no atendimento das necessidades da população e seu reflexo na longevidade como parte do processo não são mensuradas.

\subsubsection{Aplicações de técnicas de eficiência com Índice de Desenvolvimento Humano (IDH) e gasto público em saúde}

Estudos globais e locais que analisam a eficiência dos gastos públicos em saúde utilizam fontes de dados públicos, indicadores, índices, ou elaboram novos índices para esta análise. Assim, apresenta-se uma breve revisão sobre algumas aplicações de técnicas de eficiência com o IDH e os gastos públicos em saúde. Cabe destacar, a importância de uso de um indicador como o IDH em uma construção de análise de eficiência, primeiramente porque ele é uma medida global e traz confiabilidade aos estudos devido a sua consolidação, e segundo, pela oportunidade de replicação de um estudo em outras localidades.

O IDH foi elaborado por Mahbub ul Haq e Amartya Sen (Prêmio Nobel de Economia - 1998) e divulgado pela primeira vez no Relatório de Desenvolvimento Humano (Human Development Report) em 1990, com o objetivo de oferecer um contraponto à dimensão econômica Produto Interno Bruto (PIB) per capita (Atlas Brasil, 2017). O IDH é uma medida resumida do progresso em longo prazo em três dimensões básicas do desenvolvimento humano: renda, educação e saúde (Prasetyo \& Zuhdi, 2013). No Brasil, a elaboração do IDH é realizada pelo Programa das Nações Unidas para o Desenvolvimento (PNUD), pelo Instituto de Pesquisa Econômica Aplicada (IPEA) e pela Fundação João Pinheiro (FJP). Conforme o site do Atlas Brasil (2017) o IDH Global é composto pela média geométrica de três dimensões (Índice de Desenvolvimento Humano Municipal - IDH-M): fator Longevidade: expectativa de vida ao nascer; fator Educação: escolaridade da população adulta (peso 1) e fluxo escolar da população jovem (peso 2); e fator Renda: Renda per capita. Sendo representado pela fórmula:

$$
\text { DH Global }=\sqrt[3]{I D H-M \text { Longevidade } x I D H-M \text { Educação } x I D H-M \text { Renda }}
$$

Assim, tanto o IDH Global, como os três IDH-M resultam em um número de 0 a 1, que quanto mais próximo de 1, melhor será seu valor. As faixas do desenvolvimento humano são: muito baixo $(0$ a 0,49$)$, baixo $(0,5$ a 0,599$)$, médio $(0,6$ a 0,699), alto (0,7 a 0,799), e muito alto (acima de 0,8$)$.

Alguns estudos que utilizaram o IDH ou suas dimensões ou a expectativa de vida como variável na análise de eficiência dos gastos públicos em saúde e na eficiência do município, como pode ser visto em: Maciel et al. (2009), Bikis (2011), Rahmayanti e Horn (2011), Poker Jr. e Crozatti (2013), Prasetyo e Zuhdi (2013), Araújo Neto (2016) e Giménez, Ayvar-Campos e Navarro-Chávez (2017). As técnicas utilizadas nestes estudos foram: DEA, dados em painel e SFA.

Além destes estudos que utilizaram o IDH ou suas dimensões ou a expectativa de vida como output, outros estudos analisam a relação dos resultados de eficiência com este, como pode ser visto nos estudos de Mazon (2012) e Passoni (2014). O primeiro, após analisar a eficiência dos municípios de Santa Catarina, relacionou os resultados do DEA com o IDH-M fator Longevidade, identificando que os municípios com maiores despesas foram os que obtiveram maior crescimento deste índice. Da mesma forma, o segundo estudo relacionou os resultados dos municípios eficientes de São Paulo com o IDH, e verificou que o nível médio de eficiência tende a crescer quando o IDH-M em cada região administrativa aumenta. 


\section{Metodologia}

O estudo conta com a coleta e tratamento dos dados, a aplicação do modelo DEA e elaboração do predito (eficiência esperada com o tratamento das variáveis intervenientes) para o período de 2000 a 2009. O modelo DEA selecionado foi o VRS orientado aos outputs, uma vez que, busca-se um aumento na variação do Índice de Desenvolvimento Humano Municipal (IDH-M) fator longevidade sem que haja uma redução dos gastos em saúde e saneamento nos municípios estudados. Este modelo contou com a seleção de um input e um output, sendo o gasto público em saúde e saneamento per capita (GPSpc) e a variação do IDH-M fator longevidade (Var_IDHML) respectivamente. Os dados selecionados cobriram período de 10 anos e a amostra foi de 399 municípios paranaenses, mas 36 municípios foram excluídos por não disporem de dados de input, restando então 363 municípios. Assim, os dados foram coletados e transformados, conforme descrições na Tabela 2. A variável de input foi relativizada per capita para remover o efeito do tamanho do município, sendo desnecessária a aplicação de métodos de agrupamento.

Tabela 2. Resumo das Etapas de Transformação do Input e Output.

\begin{tabular}{lll}
\hline \multicolumn{1}{c}{ Variável } & \multicolumn{1}{c}{ Período/Fonte } & \multicolumn{1}{c}{ Etapas } \\
\hline $\begin{array}{l}\text { Input: GPSpc (Gasto público em } \\
\text { saúde e saneamento per capita) }\end{array}$ & FINBRA (2017) & 1 - Coleta dos dados; \\
& & 3 - Deflação dos valores a preços correntes de 2009 pelo IGP-DI; \\
& & 4 - Gasto total dividido pela população. \\
\hline Output: Var_IDHML & 2000 e 2010/ & 1 - Coleta dos dados; \\
& Atlas Brasil (2017) & 2 - Subtração do IDH-M fator Longevidade 2010 do IDH-M fator \\
& & Longevidade 2000 \\
\hline
\end{tabular}

Nota: Dados coletados nas fontes e tratados pelos autores, conforme descrito nas etapas. Fonte: Autores.

Na sequência foram selecionadas 45 variáveis intervenientes (Figura 1 - Apêndice), organizadas em seis grupos: Econômico (ECO), Geografia e População (GEO), Estrutura (EST), Saúde (SAU), Mortalidade (MOR) e Educação (EDU), para cálculo da eficiência observada (predito) através de Regressão Linear Múltipla (RLM). A estrutura do modelo DEA (input, DMU e output) e as variáveis intervenientes estão representadas na Figura 2. A parte interna da Figura 2 representa o modelo de mensuração da eficiência e a parte externa representa os grupos das variáveis que podem interferir na eficiência dos municípios.

Figura 2. Modelo DEA e Variáveis Intervenientes.



Nota: Ilustração do modelo DEA aplicado no estudo, com as variáveis de entrada e saída, e os grupos de variáveis intervenientes (Figura 1 Apêndice) aplicadas na RLM. Fonte: Autores.

Na RLM foi utilizada a função stepwise (Stata) para seleção das variáveis intervenientes, pois esta função seleciona as 
melhores variáveis independentes, com base no coeficiente de correlação e na significância estatística (Hair et al., 2010). Logo após foi gerado o Fator de Inflação da Variância (Variance Inflation Factor - VIF), que indica o grau de colinearidade ou multicolinearidade entre as variáveis independentes e tem um limite de corte no valor de 10 (Hair et al., 2010). As variáveis selecionadas serão as que apresentarem significância estatística (p-valor) abaixo de 5\% e VIF abaixo de 3.

Para desenvolvimento das etapas metodológicas, análise dos resultados e a elaboração da conclusão foram utilizadas as planilhas eletrônicas do Microsoft Excel (Windows 10), o software estatístico Stata (versão 14.1) e o programa de cartomática Philcarto.

\section{Resultados e Discussão}

\subsection{Análise dos dados}

Através da análise descritiva dos dados de input e output do modelo DEA, visualiza-se a disparidade nos gastos públicos em saúde e saneamento e na Variação do IDH-M fator Longevidade nos 363 municípios paranaenses. O gasto público em saúde e saneamento per capita médio foi de $R$ \$2.575,58; o valor mínimo foi de $R \$ 637,72$ (Ibaiti) e o máximo de R\$ 12.067.05 (Itaipulândia). Dentre o total de municípios estudados, 142 gastaram acima da média e 221 abaixo da média.

Os municípios de Itaipulândia, Pato Bragado e Entre Rios do Oeste são os três que mais gastaram em saúde e saneamento no período estudado, entretanto esses municípios fazem parte dos 15 municípios paranaenses que recebem royalties da Usina Hidrelétrica de Itaipu. Sendo assim, recebem mais recursos para investimento em saúde e saneamento, contando ainda com uma população de até sete mil habitantes cada, fazendo com que o valor per capita aumente. Os municípios listados entre os com menores gastos têm populações entre 13 e 183 mil habitantes, com exceção do município de Abatiá (8.259 habitantes), indicando que o fator populacional pode ser um limitante de recursos a serem aplicados.

Nos municípios estudados, a Variação do IDH-M fator Longevidade de 2000 e 2010 em média foi de 0,07; o valor mínimo foi de 0,027 (Nova Prata do Iguaçu) e máximo foi de 0,105 (Jundiaí do Sul). Dentre os 20 municípios com maiores variações de IDH-M fator Longevidade, dezesseis deles obtiveram valores acima de 0,1; com exceção de Rolândia, Tuneiras do Oeste e Laranjeiras do Sul. Já os 20 municípios que tiveram as menores variações, ficaram abaixo de 0,037. Assim, 185 municípios tiveram Variação do IDH-M fator Longevidade acima da média; 8 na média e 170 abaixo da média.

Os três municípios que se diferenciam na análise dos gastos e na variação do IDH-M fator Longevidade são: Entre Rios do Oeste, Guaraporema e Laranjeiras do Sul. Os dois primeiros estão classificados entre os municípios com os maiores gastos, mas Entre Rios do Oeste está também classificado como um dos que tem a menor Var_IDHML, ao contrário de Guaraporema, que tem maior variação. O terceiro está classificado entre os 20 municípios com menores gastos e com maiores Var_IDHML. Analisando os dados de input e output dos municípios que são sedes das Regionais de Saúde paranaenses, verifica-se que também não há um padrão nos gastos realizados proporcionais à variação do IDHM-L.

A média dos gastos realizados pelos municípios sedes das Regionais de Saúde foi de $\mathrm{R} \$ 2.390,45$ e a média da Variação do IDH-M fator Longevidade foi de 0,056; ambas estão abaixo da média geral. Então, entre esses municípios sedes, os 5 que tiveram os maiores gastos, entre $\mathrm{R} \$ 3.500,00$ e $\mathrm{R} \$ 4.800,00$; foram: Londrina, Pato Branco, Maringá, Curitiba e Umuarama, já os 5 menores gastos, até R \$1.500,00; foram realizados pelos municípios sedes de: Irati, Ivaiporã, União da Vitória, Jacarezinho e Guarapuava. O município sede de Regional de Saúde com maior Var_IDHML foi Telêmaco Borba, seguido de Irati e Jacarezinho, acima de 0,085; e os que tiveram uma variação abaixo de 0,04 foram: Guarapuava, Ponta Grossa e Umuarama.

\subsection{Análise dos resultados DEA-VRS}

O modelo DEA aplicado resultou em cinco DMUs eficientes e 258 ineficientes (Figura 3). Os municípios que 
atingiram a fronteira da eficiência DEA (100\%) foram: Ibaiti, Iretama, Jundiaí do Sul, Laranjeiras do Sul e Telêmaco Borba. As DMUs que se aproximaram da eficiência foram os municípios de: Paulo Frontin, Boa Ventura de São Roque, Nova Aurora, Ivaí, Teixeira Soares e Querência do Norte, com resultados que variaram entre 99,05\% a 99,85\%.

Figura 3. Mapa de Eficiências DEA.

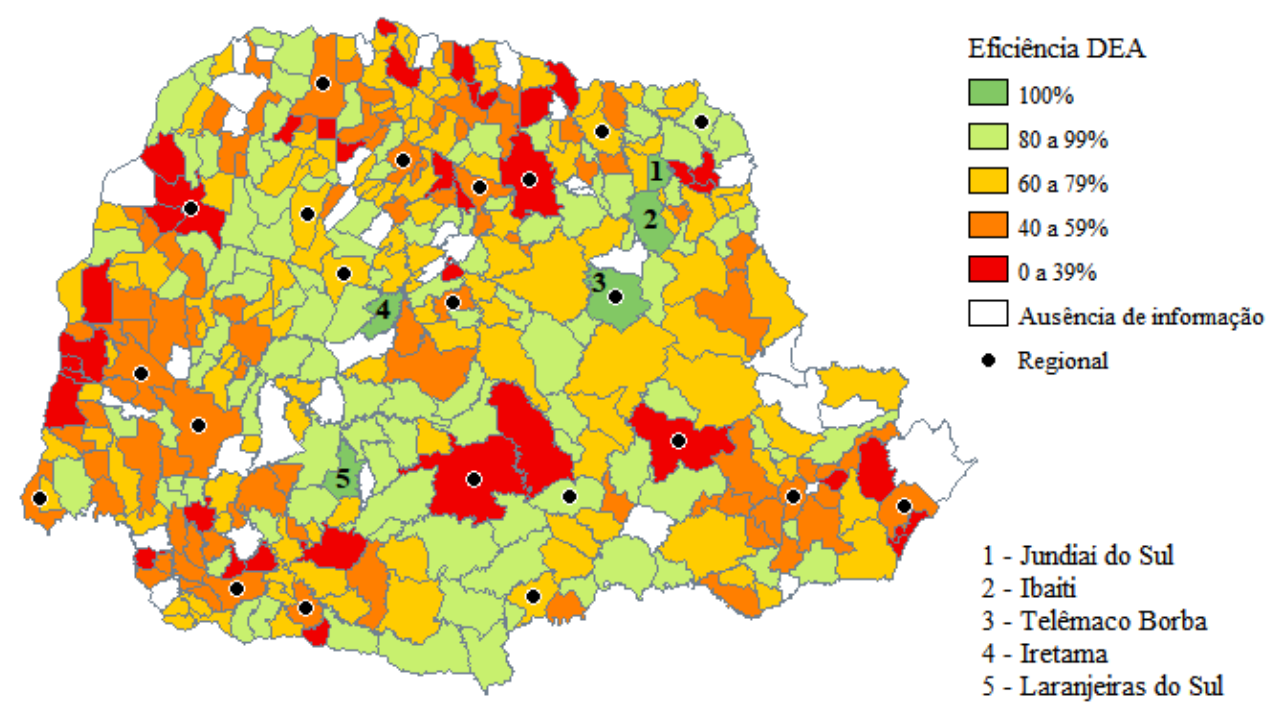

Nota: Elaborado no Philcarto com as eficiências resultadas no modelo DEA aplicado no Stata. Fonte: Autores.

Os municípios eficientes de Laranjeiras do Sul e Ibaiti foram classificados entre os 20 municípios que realizaram os menores gastos per capita em saúde, e os municípios de Telêmaco Borba, Laranjeiras do Sul, Iretama e Jundiaí do Sul foram classificados entre os 20 municípios que obtiveram as maiores Var_IDHML. E os municípios que apresentaram as piores taxas de ineficiência (abaixo de 30\%) foram: Nova Prata do Iguaçu, Centenário do Sul, Terra Roxa, Mirador e Xambrê. Apenas $21 \%$ dos 363 municípios paranaenses obtiveram ineficiências abaixo de 50\%.

A Figura 3 mostra a distribuição das eficiências e ineficiências obtidas por cada um dos municípios paranaenses. As ineficiências com valores abaixo de 39\% distribuíram-se em 38 municípios, entre 40 e 59\% em 89 municípios, entre 60\% e 79\% em 122, e entre $80 \%$ e 99,85\% em 109 municípios. Aproximadamente $65 \%$ dos municípios estudados obtiveram escores de ineficiência acima de $60 \%$.

Conforme mencionado anteriormente, o município sede da $21^{\text {a }}$ Regional de Saúde de Telêmaco Borba foi um dos 5 eficientes no modelo DEA aplicado. Ressalta-se que outros municípios sedes de Regional de Saúde ficaram próximos a eficiência DEA, como os municípios de Irati e Jacarezinho. Os outros municípios sede das demais Regionais de Saúde obtiveram escores de ineficiência abaixo de $80 \%$, sendo que mais da metade esteve abaixo de $50 \%$.

\subsection{Análise dos fatores intervenientes e eficiência esperada (predito)}

Os fatores intervenientes ao ambiente de gestão dos gastos públicos em saúde e saneamento podem afetar a eficiência resultada dos municípios na metodologia DEA, tanto positivamente como negativamente. Para identificar esses fatores foram selecionadas 45 possíveis variáveis exógenas à gestão pública (Tabela 3), testadas em estudos precursores a este e outras baseadas nos determinantes sociais. Assim, na RLM, a eficiência DEA (EfiDEA) foi a variável dependente e as 45 variáveis intervenientes foram as variáveis independentes, calculadas com as funções stepwise e VIF, pois uma seleciona o melhor conjunto de variáveis e a outra apresenta a ausência ou presença de multicolinearidade. 
Research, Society and Development, v. 11, n. 3, e2211326164, 2022

(CC BY 4.0) | ISSN 2525-3409 | DOI: http://dx.doi.org/10.33448/rsd-v11i3.26164

Tabela 3. Variáveis Intervenientes.

\begin{tabular}{|c|c|c|c|}
\hline Grupo & Variável & Descrição & Sinal esperado \\
\hline \multirow{3}{*}{$\mathrm{ECO}$} & Var $\mathrm{DHMR} \mathrm{R}^{\mathrm{a}}$ & Variação do IHM-Renda. & Positivo \\
\hline & Var Rendapc ${ }^{c}$ & Variação da Renda per capita. & Positivo \\
\hline & ProBolsaFam ${ }^{c}$ & Pessoas cadastradas no Programa Bolsa Família per capita. & Positivo \\
\hline \multirow{3}{*}{ GEO } & PopRur ${ }^{c}$ & Percentual de população rural. & Negativo \\
\hline & DistCap $^{c}$ & Distância da capital em quilômetros. & Negativo \\
\hline & DistReg ${ }^{c}$ & Distância da Regional de Saúde. & Negativo \\
\hline \multirow{4}{*}{ EST } & PopEnerg ${ }^{2}$ & Variação do percentual da população com rede elétrica. & Positivo \\
\hline & PopColix ${ }^{a}$ & Variação do percentual da população com coleta de lixo. & Positivo \\
\hline & PopAgua ${ }^{a}$ & Variação do percentual da população com água encanada. & Positivo \\
\hline & PopBan $\mathrm{Ag}^{\mathrm{a}}$ & Variação do percentual da população com banheiro e água. & Positivo \\
\hline \multirow{24}{*}{$\mathrm{SAU}$} & Equipamentos ${ }^{b}$ & Média de equipamentos. & Positivo \\
\hline & HosplMunpc ${ }^{b}$ & Média de hospitais municipais per capita. & Positivo \\
\hline & HospPrivpc $^{b}$ & Média de hospitais privados per capita. & Positivo \\
\hline & HospTot ${ }^{b}$ & Média de hospitais totais per capita. & Positivo \\
\hline & IDSUS ${ }^{\mathrm{b}}$ & Nota do IDSUS 2010. & Positivo \\
\hline & LeitosObstpc ${ }^{b}$ & Média de leitos obstétricos per capita. & Positivo \\
\hline & Leitospc $c^{b}$ & Média de leitos totais per capita. & Positivo \\
\hline & NroAmbu ${ }^{b}$ & Número de ambulatórios médicos. & Positivo \\
\hline & NroFunEst ${ }^{b}$ & Número de funcionários estaduais. & Positivo \\
\hline & NroFunFed ${ }^{b}$ & Número de funcionários federais. & Positivo \\
\hline & NroFunMun ${ }^{b}$ & Número de funcionários municipais. & Positivo \\
\hline & NroFunPriv ${ }^{b}$ & Número de funcionários privados. & Positivo \\
\hline & NroFunTot ${ }^{b}$ & Número de funcionários total. & Positivo \\
\hline & NroOdonto ${ }^{b}$ & Número de salas de odontologia. & Positivo \\
\hline & ProdAmbu ${ }^{b}$ & Quantidade de produção ambulatorial. & Positivo \\
\hline & ProSauFampc ${ }^{b}$ & Média de pessoas atendidas pelo Programa Saúde na Família per capita. & Positivo \\
\hline & VigEpidpc ${ }^{b}$ & Equipes de Vigilância Epidemiológica per capita. & Positivo \\
\hline & NroInter ${ }^{b}$ & Número de internamentos. & Negativo \\
\hline & NroDiasInter ${ }^{\mathrm{b}}$ & Número de dias de internamento. & Negativo \\
\hline & Óbitos ${ }^{\mathrm{b}}$ & Óbitos hospitalares. & Negativo \\
\hline & TxMortInter ${ }^{\mathrm{b}}$ & Taxa de mortalidade hospitalar. & Negativo \\
\hline & HFedDummy ${ }^{\mathrm{b}}$ & Dummy Município possui Hospital Federal. & Negativo \\
\hline & HEstDummy ${ }^{b}$ & Dummy Município possui Hospital Estadual. & Negativo \\
\hline & RegDummy ${ }^{\mathrm{d}}$ & Dummy Municipio é Regional de Saúde. & Negativo \\
\hline \multirow{7}{*}{ MOR } & MortHomnec & Mortalidade causada por homicidios total per capita. & Negativo \\
\hline & MortTranspc ${ }^{c}$ & Mortalidade causada por acidente de trânsito per capita. & Negativo \\
\hline & Homic Jovens $^{c}$ & Mortalidade causada por homicídio em jovens per capita. & Negativo \\
\hline & CapIX ApCirc ${ }^{b}$ & Mortalidade causada por doenças do aparelho circulatório per capita & Negativo \\
\hline & CapI Neop ${ }^{b}$ & Mortalidade causada por neoplasias per capita. & Negativo \\
\hline & $\mathrm{Cap} X \mathrm{XX} \mathrm{Acid}^{\mathrm{b}}$ & Mortalidade causada por acidentes e quedas per capita. & Negativo \\
\hline & CapX ApResp ${ }^{b}$ & Mortalidade causada por doenças do aparelho respiratório per capita. & Negativo \\
\hline \multirow{4}{*}{ EDU } & Var $\Pi \cap \mathrm{MMF}^{\mathrm{a}}$ & Variação do IDHM-Educação. & Positivo \\
\hline & Var TxAnalfa $14^{\mathrm{a}}$ & Variação da Taxa de Analfabetismo até 14 anos de idade. & Positivo \\
\hline & Var TxAnalfa $18+^{\mathrm{a}}$ & Variação da Taxa de Analfabetismo acima dos 18 anos de idade. & Positivo \\
\hline & Var EnsSun ${ }^{a}$ & Variação da Taxa de Pessoas com Ensino Superior. & Positivo \\
\hline
\end{tabular}

Nota: Variáveis coletadas nos sites do a Atlas Brasil (2017), ${ }^{\mathrm{b}}$ DataSUS (2017), ${ }^{\mathrm{c}}$ Instituto de Pesquisa Econômica Aplicada (2017) e ${ }^{\mathrm{d}}$ Secretaria Estadual de Saúde do Paraná (2016), para aplicação da RLM com a eficiência para identificar os fatores intervenientes. Fonte: Autores.

Como resultado, os fatores interventores que justificam as eficiências resultadas no DEA foram: Var_IDHME, CapII_Neopc, DistCap e RegDummy. Das quatro variáveis, três delas apresentaram p-valores abaixo de 5\%, apenas a variável 
CapII_pc resultou em um p-valor de 6,5\%, entretanto ela também foi considerada no cálculo do predito. O R2 Ajustado foi de 6,3 ; indicando que estas quatro variáveis explicam 6,3\% das eficiências e ineficiências obtidas pelos municípios no DEA. O VIF médio foi de 1,17; com valor muito abaixo do corte de 10.

O sinal dos coeficientes indica a relação da variável na eficiência, se será positiva ou negativa. Desta forma, o sinal positivo no coeficiente da Variação do IDH-M fator Educação (Var_IDHME) indica que quanto maior for este valor, maior será a eficiência da DMU. O IDH-M fator Educação mede o nível educacional dos habitantes do município, assim, com uma população com maior escolarização, pode-se ter acesso a mais conhecimento (formação), possibilidade de renda maior, condições de adquirir serviços de saúde privados, reduzindo os custos municipais em saúde pública ou ampliação dos serviços, que, consequentemente, podem aumentar a longevidade.

Ao contrário disso, os coeficientes negativos das variáveis Distância da Capital (DistCap), Dummy de Regional de Saúde (RegDummy) e Mortalidade causada por Neoplasias - Capítulo II da CID 10 per capita (CapII_pc), indicam que, quanto menor forem esses valores, maior será a eficiência predita. Essas variáveis reduzem a longevidade da população, uma vez que: a maior distância da capital reduz o acesso aos tratamentos especializados, que não estão disponíveis em todos os municípios ou nos municípios sedes das respectivas Regionais de Saúde. No Paraná, os hospitais estaduais especializados em hanseníase, tisiologia, infectologia, psiquiatria, reabilitação e infantil, estão situados na capital, Curitiba, ou em cidades limítrofes à capital (Secretaria Estadual de Saúde, 2016). Dentre as 22 Regionais de Saúde, apenas 11 possuem hospitais regionais para tratamento geral: Paranaguá, Curitiba, Francisco Beltrão, Paranavaí, Jacarezinho, Telêmaco Borba, Ponta Grossa, Cascavel, Maringá, Londrina e Guarapuava. A espera pela vaga no hospital da capital, a disponibilidade do município no transporte do paciente e outros fatores, podem causar um aumento das taxas de mortalidade, devido ao retardo no tratamento.

O município ser sede de Regional de Saúde pode fazer com que ele não seja eficiente, pois os gastos públicos em saúde e saneamento destinados a população local acabam por se dividir com os demais pacientes dos municípios desta ou, ainda, de outras regionais (fenômeno do rent-seeking).

As neoplasias são a segunda maior causa de mortalidade no estado do Paraná, ficando atrás apenas das doenças causadas pelo aparelho respiratório. Assim, a distância de um centro especializado para tratamento, o alto custo do tratamento e a falta de exames preventivos, pode gerar complicações no quadro das neoplasias e fazer com que a taxa de mortalidade aumente.

O predito é a eficiência esperada para o município em relação ao tratamento, ou redução da interferência dos fatores intervenientes, através de ações direcionadas dos gestores públicos. Assim, compara-se a eficiência esperada com a observada, onde identifica-se a diferença entre ambas, demonstrando quais municípios conseguiram tratar ou dirimir a influência dos fatores intervenientes na gestão do gasto público em saúde e saneamento per capita nos municípios frente a Var_IDHML.

Assim, a Tabela 4 mostra a eficiência observada (EfiDEA) e a eficiência esperada (EfiPRED) para os 10 municípios com as maiores e menores eficiências esperadas. Dentre o resultado geral, as maiores eficiências esperadas foram para os municípios de Goioxim, Itaperuçu e Campo Magro, ambos na faixa dos 78\%, demonstrando que não era esperada eficiência para nenhum dos municípios paranaenses estudados. As menores eficiências esperadas foram para os municípios de Londrina, Maringá e Cascavel, que ficaram abaixo de $50 \%$ de eficiência esperada. 
Tabela 4. Municípios com as Maiores e Menores Eficiências Esperadas.

\begin{tabular}{|c|c|c|c|c|c|}
\hline \multicolumn{3}{|c|}{10 Municípios com maiores EfiPRED } & \multicolumn{3}{|c|}{10 Municípios com menores EfiPRED } \\
\hline Município & EfiDEA & EfiPRED & Município & EfiDEA & EfiPRED \\
\hline Goioxim & $84,8 \%$ & $78,5 \%$ & Londrina & $39,0 \%$ & $47,2 \%$ \\
\hline Itaperuçu & $97,9 \%$ & $78,4 \%$ & Maringá & $46,7 \%$ & $47,4 \%$ \\
\hline Campo Magro & $72,6 \%$ & $78,0 \%$ & Cascavel & $42,0 \%$ & $47,9 \%$ \\
\hline Piraquara & $48,8 \%$ & $77,0 \%$ & Pato Branco & $40,0 \%$ & $50,8 \%$ \\
\hline Rio Branco do Ivaí & $77,1 \%$ & $76,6 \%$ & Campina Grande do Sul & $56,0 \%$ & $51,6 \%$ \\
\hline São José dos Pinhais & $59,3 \%$ & $76,5 \%$ & Umuarama & $31,4 \%$ & $51,6 \%$ \\
\hline Pontal do Paraná & $35,2 \%$ & $76,4 \%$ & Toledo & $55,4 \%$ & $52,1 \%$ \\
\hline Ariranha do Ivaí & $87,6 \%$ & $76,3 \%$ & Campo Mourão & $61,9 \%$ & $52,7 \%$ \\
\hline Fazenda Rio Grande & $84,7 \%$ & $76,3 \%$ & Paranavaí & $40,8 \%$ & $53,2 \%$ \\
\hline Antônio Olinto & $82,9 \%$ & $76,2 \%$ & Foz do Iguaçu & $40,0 \%$ & $53,8 \%$ \\
\hline
\end{tabular}

Nota: Resultados da aplicação da RLM no Stata, com as eficiências resultadas e as variáveis intervenientes. Fonte: Autores.

Ao analisar as eficiências DEA nos municípios com os maiores escores esperados (EfiPRED), percebe-se que, em relação a EfiDEA, alguns municípios conseguiram ter escores de ineficiência maiores, ou seja, conseguiram dirimir os impactos dos fatores intervenientes, como é o caso dos municípios de: Itaperuçu, Ariranha do Ivaí, Goioxim, Fazenda Rio Grande, Antônio Olinto e Rio Branco do Ivaí. O mesmo ocorre para alguns municípios que estão entre os 10 com as menores EfiPRED: Campina Grande do Sul, Campo Mourão e Toledo. Ressalta-se que, nove dos dez municípios classificados com menor EfiPRED são sedes de Regionais de Saúde, com exceção de Campina Grande do Sul.

\section{Considerações Finais}

Este estudo corrobora com os resultados de outros estudos globais, no sentido de que aumentar o valor dos gastos em saúde e saneamento acima de um determinado valor, não implica um município eficiente no aumento da longevidade dos munícipes. Os municípios eficientes foram aqueles que apresentaram próximos à média e alta variação da longevidade, entretanto aqueles com maiores gastos não foram eficientes, mesmo tendo variação da longevidade alta. Ou seja, embora consigam promover melhorias na expectativa de vida, não o fazem de forma mais eficiente.

A disparidade dos gastos públicos em saúde e saneamento realizados nos municípios estudados mostrou uma variação de quase 20 vezes entre o menor e o maior gasto realizado. A disparidade e a exorbitância dos gastos podem ser causadas e justificadas por alguns fatores, como: a origem dos recursos, obrigatoriedade legal da aplicação de $15 \%$ sobre as receitas municipais em saúde, tamanho da população municipal, corrupção e o desperdício.

Presume-se uma relação entre esses fatores, por exemplo: alguns municípios arrecadam maiores valores que outros (seja por royalties ou pela instalação de grandes indústrias), fazendo com que gastem mais, independentemente do tamanho da população, dada a obrigatoriedade da aplicação de 15\% das receitas com impostos por parte dos municípios em saúde, como visto no neste estudo. Alguns gestores podem fazer uso da obrigatoriedade do gasto para desperdiçar e aplicar de forma irregular (corrupção) os recursos públicos em saúde.

Um dos achados diz respeito ao fenômeno do rent-seeking, evidenciado pela significância estatística do parâmetro identificador do município como sede de Regional de Saúde. Presumindo-se que este ocorra pelo uso dos serviços de saúde nas Regionais de Saúde por parte dos municípios de competência desta regional.

A adoção de práticas de gestão eficientes no gasto público em saúde e saneamento poderia aumentar a longevidade, uma vez que, com a adoção de políticas públicas que atendam as necessidades de saúde da população, o controle e identificação de fatores intervenientes e a economicidade do dinheiro público, os resultados dos recursos alocados para a saúde 
e saneamentos nos municípios paranaenses pode ser mais eficiente, prestando um serviço de qualidade superior à população.

Dessa forma, a principal contribuição desse estudo reside nos resultados e análises elaboradas, pois podem auxiliar o gestor público na tomada de decisão sobre o gasto público em saúde e saneamento. Como ainda não havia estudos elaborados com esta temática para este estado, os municípios eficientes podem servir de base (benchmarks) para os municípios que foram identificados como ineficientes.

O estudo identificou nas variáveis disponíveis que duas dentre as 45 variáveis selecionadas ainda não haviam sido testadas ou resultaram em significância estatística em outros estudos: o município ser sede de Regional de Saúde e o percentual de incidência de neoplasias na população. Assim, o município ser sede de Regional de Saúde afeta negativamente em sua eficiência, devido ao comportamento rent-seeking, causado pela utilização dos serviços de saúde. E a incidência de neoplasias nos munícipes causa aumento dos gastos, seja com tratamento ou transporte, indicando assim, a importância de políticas públicas preventivas, que além de serem menos onerosas, promovem a longevidade da população.

As limitações encontradas na elaboração do estudo foram a falta de dados, devido a 36 municípios não terem relatado seus gastos em saúde e saneamento em um ou mais períodos; a qualidade e a efetividade dos serviços públicos em saúde prestados nos municípios estudados não foram avaliadas; e também não foram encontradas variáveis com significância estatística elevada que justificassem as eficiências resultadas pelos municípios estudados. Os avanços tecnológicos, os novos diagnósticos e medicamentos para tratamento ou prevenção de doenças não foram considerados no estudo, uma vez que estes também colaboram para o aumento da longevidade na população, mas são mais difíceis de serem mensurados.

Enfim, para trabalhos futuros recomenda-se a aplicação do modelo em outros períodos comparando as eficiências, ampliação da amostra para municípios dos demais estados brasileiros ou ainda para municípios de outros países, haja visto que, as variáveis utilizadas no modelo DEA-VRS deste estudo estão disponíveis para diversos países. Recomenda-se ainda a aplicação do método de análise de dados em painel nos dados de input e output, antes do cálculo da DEA, para identificar a defasagem e o efeito do gasto público em saúde e saneamento no período a ser estudado.

\section{Agradecimentos}

A autora Vanderléia de Souza da Silva agradece à Coordenação de Aperfeiçoamento de Pessoal de Nível Superior (CAPES) pelo apoio financeiro.

\section{Referências}

Andersen, B., \& Pettersen, P.G. (1996). The benchmarking handbook: step-by-step instructions. Chapman \& Hall.

Araujo Neto, L. M. de. (2016). Eficácia, eficiência e produtividade dos gastos públicos municipais no Brasil. Dissertação (Mestrado). Universidade de Brasília - UNB.

Atlas Brasil. (2017). O IDHM - Atlas do Desenvolvimento Humano no Brasil. http://atlasbrasil.org.br/2013/pt/o_atlas/idhm/

Banker, A. R. D., Charnes, A., \& Cooper, W. W. (1984). Some Models for Estimating Technical and Scale Inefficiencies in Data Envelopment Analysis. Management Science, 30(9), 1078-1092.

Bikis, J. (2011). Efficiency of the Welfare State: a comparative approach using Data Envelopment Analysis. University of Texas. https://doi.org/10.1111/j.1467-6435.1999.tb00232.x

Buss, P. M. (2000). Promoção da saúde e qualidade de vida. Ciência \& Saúde Coletiva, 5(1), 163-177. https://doi.org/10.1590/S1413-81232000000100014

Campos, M. S., Fernández-Montes, A., Gavilan, J. M., \& Velasco, F. (2016). Public resource usage in health systems: a data envelopment analysis of the efficiency of health systems of autonomous communities in Spain. Public Health, 138, 33-40. https://doi.org/10.1016/j.puhe.2016.03.003

Carrara, B. S., \& Ventura, C. A. A. (2012). A saúde e o desenvolvimento humano. Saúde \& Transformação Social, 3(4), 89-96. http://incubadora.periodicos.ufsc.br/index.php/saudeetransformacao/article/view/1484/2247

Charnes, A., Cooper, W. W., \& Rhodes, E. (1978). Measuring the efficiency of decision making units, short communication. European Journal of Operational Research, 3, 339. https://doi.org/http://dx.doi.org/10.1016/0377-2217(78)90138-8 
Governo do Estado do Paraná. (2017). Etnias - Estado do Paraná. http://www.cidadao.pr.gov.br/modules/conteudo/conteudo.php?conteudo=77

Coelli, T. J., Rao, D. S. P., O’Donnel, C. J., \& Batesse, G. E. (2005). An introduction to efficiency and productivity analysis. New York, USA: Springer.

Cooper, W. W., Seiford, L. M., Zhu, J., \& Hillier, F. S. (2011). Handbook on Data Envelopment Analysis. (F. S. Hillier, Org.), Springer (Second, Vol. 164). London: Springer. https://doi.org/10.1007/978-1-4419-6151-8

Cooper, W. W., Seiford, L. M., \& Tone, K. (2002). Data Envelopment Analysis: A Comprehensive Text with Models, Applications,References and DEASolver Software. Journal of Chemical Information and Modeling (Vol. 53). https://doi.org/10.1017/CBO9781107415324.004

DataSUS. (2017). Informações de Saúde. http://www2.datasus.gov.br/DATASUS/index.php?area=02

Dias, L. N. S. (2016). Fatores que impactam na corrupção e na ineficiência relacionadas à aplicação de recursos da saúde pública municipal. Universidade de Brasília, Universidade Federal da Paraíba e Universidade Federal do Rio Grande do Norte.

Dyson, R. G., Allen, R., Camanho, A. S., Podinovski, V. V, Sarrico, C. S., \& Shale, E. A. (2001). Pitfalls and protocols in DEA. European Journal of Operational Research, 132, 245-259. http://ac.els-cdn.com/S0377221700001491/1-s2.0-S0377221700001491-main.pdf?_tid=b4c7db3c-44ca-11e7-92de00000aab0f02\&acdnat=1496102486_ed35562fc8105005184bf5e47dbc5fb0

Emrouznejad, A., Parker, B. R., \& Tavares, G. (2008). Evaluation of research in efficiency and productivity: A survey and analysis of the first 30 years of scholarly literature in DEA. Socio-Economic Planning Sciences, 42(3), 151-157. https://doi.org/10.1016/j.seps.2007.07.002

Finanças do Brasil. (2017). Contas Anuais - Artigo Prefeituras e Governos. Sistema do Tesouro Nacional (STN). http://www.tesouro.fazenda.gov.br/pt_PT/contas-anuais

Giménez, V., Ayvar-Campos, F. J., \& Navarro-Chávez, J. C. L. (2017). Efficiency in the generation of social welfare in Mexico: A proposal in the presence of bad outputs. Omega (United Kingdom), 69, 43-52. https://doi.org/10.1016/j.omega.2016.08.001

Hair, J., Anderson, R., Tatham, R., \& Black, W. (2010). Multivariate data analysis (7th ed). Pearson.

Hsu, Y. C. (2013). The efficiency of government spending on health: Evidence from Europe and Central Asia. The Social Science Journal, 50(4), 665-673. https://doi.org/10.1016/j.soscij.2013.09.005

Instituto de Pesquisa Econômica Aplicada. (2017). Banco de dados regional e social. http://www.ipeadata.gov.br/Default.aspx

Lepchack, A., Lima Filho, S. S., Silva, E. O., \& Peixe, B. C. S. (2021). Análise da eficiência na utilização de recursos da saúde nos maiores municípios brasileiros. Research, Society and Development, v. 10, n. 15. http://dx.doi.org/10.33448/rsd-v10i15.22669

Liu, J. S., Lu, L. Y. Y., Lu, W. M., \& Lin, B. J. Y. (2013). A survey of DEA applications. Omega (United Kingdom), 41(5), 893-902. https://doi.org/10.1016/j.omega.2012.11.004

Maciel, V. F., Piza, C. C. de T., \& Penoff, R. N. (2009). Desigualdades regionais e bem-estar no brasil: quão eficiente tem sido a atividade tributária dos estados para a sociedade? Planejamento e Políticas Públicas, 33(2), 291-318.

Marinho, A. (2001). Estudo de eficiência em hospitais públicos e privados com a geração de rankings. Revista de Administração Pública, 32(6), 145-58. http://bibliotecadigital.fgv.br/ojs/index.php/rap/article/viewArticle/7785

Mazon, L. M. (2012). Reflexos da aplicação dos recursos financeiros públicos em saúde no desenvolvimento regional. Dissertação (Mestrado). Universidade do Contestado - Canoinhas, SC.

Novignon, J. (2015). On the efficiency of public expenditure in Sub-Saharan Africa: Does corruption and quality of public institutions matter? Munich Personal RePEc Archive, 21. http://mpra.ub.uni-muenchen.de/39195/MPRA

Ozcan, Y. A. (2008). Health care benchmarking and performance evaluation: An assessment using Data Envelopment Analysis (DEA). Springer. https://doi.org/10.1007/978-0-387-75448-2_2

Secretaria Estadual de Saúde. (2016). Plano Estadual de Saúde Paraná 2016-2019. Curitiba. http://www.saude.pr.gov.br/arquivos/File/PlanoEstadualSaude2016MioloAlt.pdf

Passoni, P. F. M. (2014). Eficiência na oferta de serviços de saúde no estado de São paulo: uma análise comparativa entre municípios selecionados. Dissertação (Mestrado). Universidade Carlos. https://repositorio.ufscar.br/bitstream/handle/ufscar/2162/PASSONI_PEDRO_2014.pdf?sequence=1\&isAllowed=y

Programa das Nações Unidas para o Desenvolvimento. (2017). O que é Desenvolvimento Humano? http://www.br.undp.org/content/brazil/pt/home/idh0/conceitos/o-que-e-desenvolvimento-humano.html

Poker Jr., J. H., \& Crozatti, J. (2013). Gastos Públicos com saúde e qualidade de vida nos municípios brasileiros: Influência na variação do IDH expectativa de vida na última década. Caderno de Finanças Públicas ESAF. www.esaf.fazenda.gov.br/assuntos/biblioteca/arquivos-gerais/cfp-n13_2013.pdf

Prasetyo, A. D., \& Zuhdi, U. (2013). The Government Expenditure efficiency towards the Human Development. Procedia Economics and Finance, 5(2012), 615-622. https://doi.org/10.1016/S2212-5671(13)00072-5

Rahmayanti, Y., \& Horn, T. (2011). Expenditure efficiency and the optimal size of government in developing countries. Global Economy and Finance Journal, $4(2), 46-59$. 
Research, Society and Development, v. 11, n. 3, e2211326164, 2022

(CC BY 4.0) | ISSN 2525-3409 | DOI: http://dx.doi.org/10.33448/rsd-v11i3.26164

Reisman, D. (2007). Health care and public policy. Edward Elgar Publishing.

Rocha, F., Duarte, J., Oliveira, P. P., Pereira, L. F. V. N., \& Gadelha, S. R. B. (2017). Are more resources always the answer? A supply and demand analysis for public health services in Brazilian municipalities. EconomiA, 18(1), 98-116. https://doi.org/10.1016/j.econ.2016.10.001

Santos, L. M., Souza Francisco, J. R., \& Gonçalves, M. A. (2016). Controle na alocação de recursos na saúde pública: uma análise nas microrregiões do sudeste brasileiro. Administração Pública e Gestão Social, 1(2), 119-130.

Seiford, L., \& Thrall, R. (1990). Recent developments in DEA: The mathematical programming approach to frontier analysis. Journal of Econometrics, 46, $7-$ 38. https://doi.org/10.1016/0304-4076(90)90045-U

World Health Organization. (2017). General Government expenditure on health as a percentage of total government expenditure. http://gamapserver.who.int/mapLibrary/Files/Maps/GenGovExpTotal_2014.png 